\title{
UV-embossed optical components for educational purposes
}

Michael Hutley

Michael C. Hutley, "UV-embossed optical components for educational purposes," Proc. SPIE 3190, Fifth International Topical Meeting on Education and Training in Optics, (8 December 1997); doi: 10.1117/12.294377

Event: Fifth International Topical Meeting on Education and Training in Optics, 1997, Delft, Netherlands 
UV Embossed optical components for educational purposes

\author{
M C Hutley \\ National Physical Laboratory \\ Teddington TW11 0LW, UK \\ MCH@NPL.CO.UK
}

Keywords: Embossing, Replication, Micro-optics, Demonstration

Abstract
UV Embossing offers the possibility to provide a range of optical components for educational purposes at very low cost.

\title{
1. Introduction
}

UV embossing is a technique for replicating surface structure with very high resolution and with high precision at relatively low cost. A layer of monomeric material is coated on a substrate, which may be rigid or flexible. The surface to be copied is impressed into the layer and since the monomer is relatively fluid it accurately adopts the form of the surface. The monomer is then polymerised by UV irradiation while still in contact with the master surface and then separated. The technique has the advantages over hot embossing that the surface is more faithfully reproduced and that the end result is harder and more durable.

Many of the components that feature in optics courses at a variety of levels either occur naturally in the form of a microsurface- relief structure or an equivalent can be produced in this form. It is possible to construct a master surface in the form of a mosaic of separate components which may be replicated together on a single plastic sheet. Individual components may then be cut out with scissors and used as required. The overall figure is of course limited by the substrate but the performance can be quite adequate to demonstrate the optical principles which they embody and can be used to build up simple systems. This is particularly true for components that are used in transmission.

The types of structure that can usefully be copied fall into three categories,

- Refracting with a surface modulation up to $50 \mu \mathrm{m}$ deep. For example micro-prism arrays, Fresnel lenses. Microlens arrays and diffusers.

- Diffracting with a modulation depth of about $1 \mu \mathrm{m}$. For example diffraction gratings, holograms, Fresnel zone plates and kinoforms.

- Sub-wavelength structures . For example motheye antireflection surfaces, polarisers and zero-orderdiffraction devices as colour filters.

\section{Experimental prototype}

We have produced a prototype mosaic of optical components built up from a variety of masters from different sources. A copy of each was first made in silicone rubber and used as a mould from which to cast a replica in epoxy resin on a square substrate of standard size. These were then cemented together to form a large array and a further rubber copy made. From this a replica was cast in epoxy resin. At the time of the meeting we demonstrated such a copy cast on a flexible plastic sheet to illustrate the form in which the UV replicas would be produced. A rigid replica has also been cast from which a nickel embossing shim has been made.

The present example contains the following items

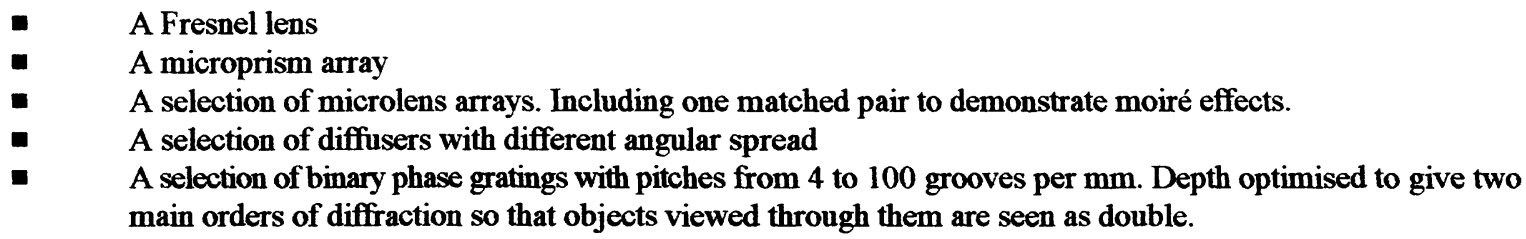


- A selection of sinusoidal spectrographic gratings with pitches between 1200 and 300 grooves per mm

- $\quad 300$ gr per mm grating strongly blazed in the visible

- Binary Fresnel zone plate showing a large number of diffracted orders. An object viewed through it is seen as multiple images of different magnifications.

- Blazed Fresnel zone plate equivalent to a 4 diopter spectacle lens

- Selection of diffracting elements to focus light into a point, a circle, a pair of concentric circles, an octagon. (Courtesy of R Smith of Imperial College)

\section{Summary}

The purpose of producing this was to demonstrate that a range of optical components from a variety of sources could be combined. If these are produced in large quantities, the cost can be very low. It then becomes practical to distribute such sheets at a price which is within the means of individual students in developed counties and within the means of educational establishments in under-developed countries. We suggest that the opportunity for students to have direct access to items such as lenses, prisms, gratings, holograms etc, would not only enhance their understanding of optics but would also help to fire their enthusiasm for the subject.

We wish to discuss the possibility that the ICO might take the initiative in designing optics kits of this type using its good influence throughout the world to obtain suitable masters, either as donations or on a commercial basis. It could then coordinate the writing and translation of handbooks which describe the experiments for which they can be used and which explain the optical principles involved. The manufacture and no doubt much of the distribution would have to be conducted on a commercial basis but it may be appropriate that a royalty be paid to the ICO in respect of its contribution.

\section{Reference}

A guide to the process and applications of UV embossing is available from the National Physical Laboratory at the above address . Measurement Good Practice Guide No 6 "UV embossed optical microstructured surfaces" By MC Hutley, N Holmes, TG Harvey and N Carter. 